\title{
Krieg und Frieden im Südkaukasus. Der ewige Konflikt um Berg-Karabach
}

\author{
Mikheil Sarjveladze
}

Angenommen: 17. Dezember 2020 / Online publiziert: 7. Januar 2021

(C) Der/die Autor(en) 2021

Zusammenfassung Der jüngste Krieg zwischen Armenien und Aserbaidschan veränderte den 26 Jahre bestehenden Status quo in der Region. Die aktuelle Veränderung des Status quo war bedingt durch das Zusammenspiel dreier Faktoren: 1) gescheiterte internationale Konfliktvermittlung, 2) die Rüstungsspirale der Konfliktparteien, 3) die Veränderung des geopolitischen Gleichgewichts durch Eingriffe der Türkei und Russlands. Im Ergebnis profitierten Moskau und Ankara von der Passivität der westlichen Akteure vor allem der EU und bauten ihren Einfluss im Südkaukasus aus.

Schlüsselwörter Berg-Karabach · Krieg · Armenien · Aserbaidschan · Russland

\section{War and Peace in the South Caucasus. The Eternal Conflict over Nagorno-Karabakh}

Abstract The recent war between Armenia and Azerbaijan changed the status quo in the region, which had existed for 26 years. The current change in the status quo was caused by the interplay of three factors: 1) the failure of international conflict mediation, 2) the conflict parties' arms race, 3) the change in geopolitical balance caused by Turkey and Russia. As a result, Moscow and Ankara benefited from the passivity of Western actors, especially the EU, and expanded their influence in the South Caucasus.

Keywords Nagorno Karabakh $\cdot$ War $\cdot$ Armenia $\cdot$ Azerbaijan · Russia

Im Rahmen eines Forschungsstipendiums der Konrad-Adenauer-Stiftung (KAS) ist der Autor Gastwissenschaftler bei der Stiftung Wissenschaft und Politik (SWP). Der Beitrag gibt ausschließlich die persönliche Meinung des Autors wieder.

Dr. M. Sarjveladze $(\bowtie)$

Stiftung Wissenschaft und Politik, Ludwigkirchplatz 3-4, 10917 Berlin, Deutschland

E-Mail: Mikheil.Sarjveladze@swp-berlin.org 


\section{Alte Probleme, neue Dynamiken}

Der Herbstkrieg 2020 zwischen Armenien und Aserbaidschan erinnert in seinem Ausmaß stark an den Karabach-Krieg von 1991 bis 1994. Zwei Unterschiede zwischen beiden Kriegen treten dennoch deutlich hervor. 1) Aserbaidschan ging dieses Mal als Sieger hervor und änderte damit den seit 1994 bestehenden Status quo zu seinen Gunsten. 2) Die Türkei als externer Akteur mischte sich neben Russland proaktiv in den Konflikt ein. Der Verlauf sowie der Ausgang des Krieges werfen vor allem die Frage auf, welche Faktoren zur Veränderung des Status quo geführt haben und warum ausgerechnet zum jetzigen Zeitpunkt? ${ }^{1}$

Um Berg-Karabach fanden bereits vor 100 Jahren blutige Auseinandersetzungen zwischen Armeniern und Aserbaidschanern (1905 und 1917-1920) statt. Im Russischen Zarenreich war Berg-Karabach dem Gouvernement von Jelisawetpol (Gandscha), der zweitgrößten Stadt im heutigen Aserbaidschan, untergeordnet. Die bergige Region wurde zu dem Zeitpunkt mehrheitlich von ethnischen Armenier*innen bewohnt, was durch die Vertreibungen aus dem Osmanischen Reich sowie durch die Ansiedlungen im Zarenreich des 19. Jahrhunderts zustande gekommen war (Halbach 2020a). Nach der Sowjetisierung der ersten armenischen und aserbaidschanischen Republiken im Jahr 1920 haben die Bolschewiki den zuvor bestehenden Status quo nicht verändert und im Jahr 1921 Berg-Karabach als autonomes Gebiet der Aserbaidschanischen Sozialistischen Sowjetrepublik zugeteilt (Quiring 2009, S. 19). Der über Jahrzehnte anhaltende Konflikt eskalierte ab 1988 im Zuge von Reformen, die der damalige Generalsekretär des Zentralkomitees der Kommunistischen Partei der Sowjetunion Michail Gorbatschow eingeleitet hatte (Quiring 2009, S. 38), und mündete in einen bewaffneten Konflikt zwischen 1991 und 1994. In diesem Krieg verloren schätzungsweise 25.000 Menschen ihr Leben und über 700.000 Aserbaidschaner*innen sowie 400.000 Armenier*innen mussten ihre Häuser verlassen (International Crisis Group 2007, S. 5). Armenien besetzte nicht nur Berg-Karabach, sondern auch sieben umliegende Bezirke, die völkerrechtlich ebenso wie das Gebiet von Berg-Karabach zu Aserbaidschan gehören.

In der Vergangenheit hinderten vor allem zwei Faktoren die Konfliktregelung: 1) Die Berg-Karabach-Frage nimmt im Prozess von Nation Building von Armeniern und Aserbaidschanern den wichtigsten Platz ein. 2) Externe Akteure, vor allem Russland, gaben sich mit dem Status quo zufrieden, blieben folglich untätig oder nutzten den Status Quo zur eigenen Einflusssicherung.

\section{Trennlinien zwischen Armenien und Aserbaidschan}

Zur wichtigsten Trennlinie gehört der nationale Diskurs in Armenien und Aserbaidschan, nach dem beide Staaten ohne Berg-Karabach nicht als Staaten vollendet seien (De Waal 2011, S. 140). In der Frage über den Status von Berg-Karabach treffen zwei Prinzipien des Völkerrechts aufeinander. Während Armenien auf seinem

\footnotetext{
1 Das Manuskript dieser Analyse wurde am 10. Dezember 2020 fertiggestellt und berücksichtigt somit die Ereignisse bis zu diesem Zeitpunkt.
} 
Selbstbestimmungsrecht der Völker besteht, beruft sich Aserbaidschan auf das Prinzip der territorialen Integrität. Die offizielle Position Bakus wurde unter anderem von vier Resolutionen des UN-Sicherheitsrates unterstützt, die die territoriale Integrität Aserbaidschans bekräftigen (UN-Sicherheitsratsresolutionen 822, 853, 874, 884). Über Jahrzehnte hinweg wurde Berg-Karabach weder von Armenien noch einem anderen Staat als unabhängiger Staat anerkannt. Die Fragen nach dem Schicksal der umliegenden Bezirke, dem finalen Status Berg-Karabachs oder der Rückkehr der Vertriebenen konnten nicht gelöst werden. Seit dem Waffenstillstand 1994 forderten Zwischenfälle 25 bis 30 Menschenleben jährlich (Smolnik und Halbach 2016, S. 70), was die Kluft noch mehr vertiefte.

\section{Internationale Konfliktvermittlung}

Seit 1992 vermittelt die Minsk-Gruppe der Organisation für Sicherheit und Zusammenarbeit in Europa (OSZE), die von drei Co-Vorsitzenden - Russland, USA und Frankreich geleitet wird. Das Mandat der OSZE im Hinblick auf die Beobachtung und Ermittlung der Waffenstillstandsverletzungen blieb allerdings äußerst beschränkt (Smolnik und Halbach 2016, S. 71). Die Lage entlang der 176 km langen Kontaktlinie in Berg-Karabach durfte die OSZE nur mit sechs Beobachter*innen inspizieren (Stöber 2020a). Durch die Mediation der Minsk-Gruppe konnte weder ein Durchbruch erreicht noch die Kriege in den Jahren 2016 und 2020 verhindert werden. Seit 2015 starben im Konflikt 1268 Militärangehörige und 96 Zivilist*innen; 58 Militärangehörige sowie 257 Zivilisten*innen wurden verwundet (International Crisis Group 2020). ${ }^{2}$ Während des Waffenganges im April 2016 starben mehr als 200 Soldat*innen auf beiden Seiten und über 200 Personen wurden verwundet (Safi 2020).

Die im Jahr 2007 von den Co-Vorsitzenden der Minsk-Gruppe entwickelten und 2009 modifizierten Madrider Prinzipien bilden seitdem die Grundlage zur Verhandlung (OSZE 2009). Eine Zustimmung der Konfliktparteien zu den Madrider Grundsätzen blieb unter anderem aus, weil Armenien nicht bereit war, besetzte Gebiete zurückzugeben und Aserbaidschan den Abzug armenischer Truppen als die Voraussetzung für die weiteren Schritte betrachtete. Die Entwicklungen seit dem RussischGeorgischen Krieg im Jahr 2008 führten zur Stärkung des technischen Charakters der OSZE-Minsk-Gruppe im Hinblick auf die Konfliktmediation (De Waal 2020). Die Rolle der OSZE als Mediator ist seitdem symbolischer geworden, weil sich beide Konfliktparteien, vor allem aber Aserbaidschan auf die Neutralität Moskaus fokussierten. Darüber hinaus profilierte sich Russland zunehmend als Schlüsselakteur und Mediator (Moskauer Deklaration 2008, Mediation während des Krieges 2016) und untergrub dadurch die Autorität der OSZE.

\footnotetext{
2 Die Statistik beinhaltet nicht die Zahl der Opfer in den Kriegen 2016 und 2020.
} 


\section{Der Berg-Karabach-Konflikt als Grund für den Rüstungswettlauf}

Der Rüstungswettlauf zwischen den Konfliktparteien resultierte in der Vergangenheit überwiegend aus der gescheiterten Konfliktmediation, wodurch die Eskalationsgefahr stetig wuchs. Laut dem Global Militarization Index (GMI) des Bonn International Center for Conversion gehören Armenien und Aserbaidschan zu den zehn Ländern mit dem höchsten Militarisierungsgrad weltweit (Mutschler und Bales 2019, S. 6). Im GMI für das Jahr 2018 belegte Armenien den 3. und Aserbaidschan den 10. Platz. Nach den Angaben des Stockholm International Peace Research Institute (SIPRI) sind zwischen 1995 und 2019 die Militärausgaben von Armenien von 52,2 Mio. US-\$ auf 673,3 Mio. US-\$ gestiegen. Im Fall von Aserbaidschan stiegen die Militärausgaben im identischen Zeitraum von 62,2 Mio. US-\$ auf 1854,2 Mio. US-\$ (SIPRI 2019). Allein im letzten Jahrzehnt gaben Aserbaidschan mehr als 24 Mrd. US-\$ und Armenien 4,7 Mrd. US-\$ für ihre Verteidigung aus. Der Anteil von Militärausgaben am BIP von Armenien betrug 2018 4,9\% und im Fall Aserbaidschans 4,0\%. Im Zeitraum von 2015 bis 2019 gehörten laut SIPRI zu Aserbaidschans größten Waffenimporteuren Israel (60\%), Russland (31\%) und die Türkei (3,2\%). Im Fall Armeniens entfielen $94 \%$ aller Waffenimporte auf Russland (SIPRI 2020).

Trotzt des Waffenembargos der OSZE seit 1992 sind es die OSZE-Länder bzw. Partnerländer selbst, die zu den größten Waffenimporteuren der Konfliktparteien zählen. Russland begründet seine Doppelrolle als gleichzeitiger Co-Vorsitzender der Minsk-Gruppe und Waffenlieferant mit Balancewahrung und verkauft Waffen an Armenien zum Vorzugspreis im Rahmen der Organisation des Kollektiven Sicherheitsvertrags (OVKS) und an Aserbaidschan zum Marktpreis (Halbach 2020b). Die Rüstungsspirale als Ergebnis des unveränderten Status Quo war dementsprechend einer der Hauptfaktoren, welcher die Konfliktentfachung beschleunigte.

\section{Der Herbstkrieg 2020}

Der Herbstkrieg 2020, der am 27. September ausbrach und bis zum 10. November andauerte, bildete seit 1994 die längste und gewaltsamste Auseinandersetzung. Laut den offiziellen Angaben sind dem Krieg 2425 armenische und 2783 aserbaidschanische Soldat*innen zum Opfer gefallen. Darüber hinaus starben mehr als 100 Zivilist*innen auf beiden Seiten und mehrere hundert wurden verwundet (BBC 2020). Menschenrechtsorganisationen wie Amnesty International und Human Rights Watch berichteten über die Anwendung verbotener Cluster- und Splittermunition durch beide Konfliktparteien (Amnesty International 2020; Human Rights Watch 2020). Vor dem Krieg lebten in Berg-Karabach circa 145.000 Menschen. Laut Angaben des Kinderhilfswerks der Vereinten Nationen (Stand 28. Oktober 2020) mussten bis zu 130.000 Personen ihre Häuser verlassen (UNICEF 2020).

Vorphase des Krieges Den Vorlauf für den Herbstkrieg 2020 bildete die militärische Auseinandersetzung im Jahr 2016, durch die Aserbaidschan dank moderner Waffen einen symbolischen Sieg errang. Dass der Konflikt zwischen Armenien und 
Aserbaidschan erneut aufflammen würde, war zu erwarten gewesen, nachdem sich beide Staaten zwischen dem 12. und 16. Juli 2020 schwere Gefechte lieferten. Die Zwischenfälle dauerten allerdings bis September an. Der Verschlechterung der Konfliktdynamik im Juli war eine relative Entspannungsphase zwischen der Samtenen Revolution in Armenien (Frühling 2018) und dem Frühjahr 2019 vorausgegangen. In dieser Phase intensivierten die Konfliktparteien ihre gegenseitige Kommunikation und die Zahl der Zwischenfälle sank deutlich (Meister 2020, S. 10-12). Letztendlich konnten die bestehenden Trennlinien aber aufgrund mangelnder Kompromissbereitschaft nicht überwunden werden. Diese negative Dynamik spiegelte sich in der offensiven Rhetorik der Konfliktparteien wider. Die Aussage des armenischen Premierministers Nikol Paschinjan im Sommer 2019, „Artsakh is Armenia, and that's it“ (zit. n. Kucera 2019), die vermutlich dem immensen innenpolitischen Druck geschuldet war, bereitete der Entspannungsphase aus aserbaidschanischer Sicht endgültig ein Ende.

Die nationale Identität Armeniens ist von dem Sieg in Berg-Karabach im Jahr 1994 und dessen symbolischer Bedeutung sehr stark geprägt. Im innenpolitischen Kontext führt die Kompromissbereitschaft in der Berg-Karabach-Frage zu einer Art politischem Selbstmord. Auch Paschinjan versuchte letztendlich diesem Schicksal zu entgehen, in dem er die zuerst versöhnliche Rhetorik in die harschen Stellungnahmen verwandelte. Aserbaidschan hingegen befand sich trotz seiner deutlichen wirtschaftlichen und militärischen Überlegenheit seit 1994 im Modus eines strategischen Wartezustands, um zu einem günstigen Zeitpunkt seine territoriale Integrität wiederherzustellen. Dieses Auseinanderdriften der Perspektiven erschwerte den Frieden zusätzlich. Noch im Dezember 2019 bezeichnete Aserbeidschans Präsident Ilham Alijew das Jahr 2019 als „,ein verlorenes Jahr im Hinblick auf die Konfliktlösung“ (Aliyev 2019, eigene Übersetzung). Die Perspektivlosigkeit zeigte sich spätestens auf der Münchener Sicherheitskonferenz im Februar 2020, auf der sich Paschinjan und Alijew mehr mit den Geschichtsstunden beschäftigten als mit den Fragen der Gegenwart oder gar der Zukunft.

Verlauf des Krieges Unmittelbar während des Krieges kam es nicht nur entlang der Konfliktlinie zu Kampfhandlungen. Auch die Städte und Dörfer auf beiden Seiten sind unter Beschuss geraten. Die Kämpfe zwischen den armenischen und aserbaidschanischen Einheiten ereigneten sich zwar auch im nördlichen Teil BergKarabachs, aber vorwiegend im Süd-Osten der Region. Bis Anfang November konnte Aserbaidschan 210 Ortschaften unter seine Kontrolle bringen (Zeit Online 2020). Die bergige Landschaft von Berg-Karabach und das Netz von Militärinfrastruktur bis zur georgischen Grenze im Norden bildete für Aserbaidschan lange Zeit ein schwer einnehmbares Territorium (Stöber 2020b). Im Herbstkrieg 2020 konnte Baku allerdings durch moderne Waffen, türkische und israelische Drohnen, Angriffe von hoher Präzision ausführen und das Festungsnetz durchbrechen. Mittels moderner Technik gelang es Aserbaidschan den geografischen Nachteil auszugleichen (Stöber 2020b), wobei die Türkei im Hinblick auf den Waffen- und Wissenstransfer eine erhebliche Rolle gespielt haben soll.

Armeniens neues sicherheitspolitisches Konzept aktiver Abschreckung, das die konventionelle Abschreckung durch Präventionsschläge auf dem Territorium des 
Angreifers vorsieht (Mediamax 2019), konnte ohne aktive russische Unterstützung und aufgrund des technischen Rückstandes nicht umgesetzt werden. Zwei Faktoren führten dazu, dass die armenische Seite am Ende umfassende Zugeständnisse machen musste: 1) die Erschöpfung der armenischen Armeeeinheiten (Paschinjan 2020) und die aufgegebene Hoffnung auf externe Unterstützung (tagesschau 2020a); 2) der Verlust der strategisch wichtigen Stadt Shusha (armenisch Shushi), die auf den Hügeln oberhalb und unweit $(14 \mathrm{~km})$ des administrativen Zentrums von BergKarabach, Stephankert, sowie auf der einzigen Verbindungsstraße liegt, die BergKarabach mit Armenien verbindet.

Vereinbarung vom 9. November Vor dem 9. November wurden drei Waffenstillstandsvereinbarungen, vermittelt durch Russland, Frankreich und die USA als Mitgliedstaaten der OSZE-Minsk-Gruppe, von den Konfliktparteien verletzt. Die Vereinbarung vom 9. November sieht außer des vollständigen Waffenstillstands vor allem folgende Punkte vor: bis zum 1. Dezember Abzug armenischer Truppen und schrittweise Rückgabe der Bezirke Agdam, Kalbadschar und Latschin an Aserbaidschan; unter aserbaidschanischer Kontrolle bleiben alle Territorien (inklusive Stadt Shusha), die Aserbaidschan zwischen dem 27. September und 10. November unter seine Kontrolle gebracht hat; Stepankert wird durch den 5 Kilometer breiten Latschin-Korridor mit Armenien (Stepankert-Goris) verbunden; es wird ein weiterer Korridor zwischen der aserbaidschanischen Exklave Nachitschewan und Aserbaidschan über das armenische Gebiet eingerichtet; zur Überwachung der Lage und Absicherung der Korridore werden 1960 russische Militärangehörige eingesetzt; Binnenvertriebene und Flüchtlinge werden unter der Aufsicht der Vereinten Nationen zurückkehren können (Presidential Executive Office 2020a). Der Vereinbarung vom 9. November folgte die Unterzeichnung des russisch-türkischen Memorandums über die Einrichtung des Monitoringzentrums zur Überwachung des Waffenstillstands mit Beteiligung von russischen und türkischen Vertreter*innen. Türkische Friedenstruppen werden allerdings im Gegensatz zu den russischen Vertretern nicht unmittelbar Berg-Karabach betreten können;

Die Vereinbarung spiegelt das Ausmaß der Niederlage Armeniens wider. Armenien verliert die Kontrolle über die sieben Bezirke um Berg-Karabach und über einen Teil des Kernterritoriums von Berg-Karabach. Proportional zur Schwächung der armenischen Position in Berg-Karabach ist Armeniens Abhängigkeit von Russland größer geworden. Aserbaidschan ist eindeutig der Sieger. Baku konnte seine territoriale Integrität zum großen Teil wiederherstellen, eine direkte Verbindung zur Nachitschewan bzw. zum Bruderstaat Türkei (Eine Nation, zwei Staaten) aushandeln und das Territorium um Stepankert faktisch einkreisen. Der Deal stärkte in erster Linie das autoritäre Regime in Baku. Allerdings nimmt auch die Abhängigkeit Aserbaidschans von Russland zu. Russische Soldat*innen werden wieder auf aserbaidschanischem Territorium stationiert sein. Darüber hinaus werfen einzelne Punkte der Verhandlungen (z. B. Korridor über Armenien, Rückkehr der Vertriebenen oder die exakte Rolle der Türkei) viele Fragen auf, die ein erhebliches Konfliktpotenzial beinhalten (Abb. 1). 


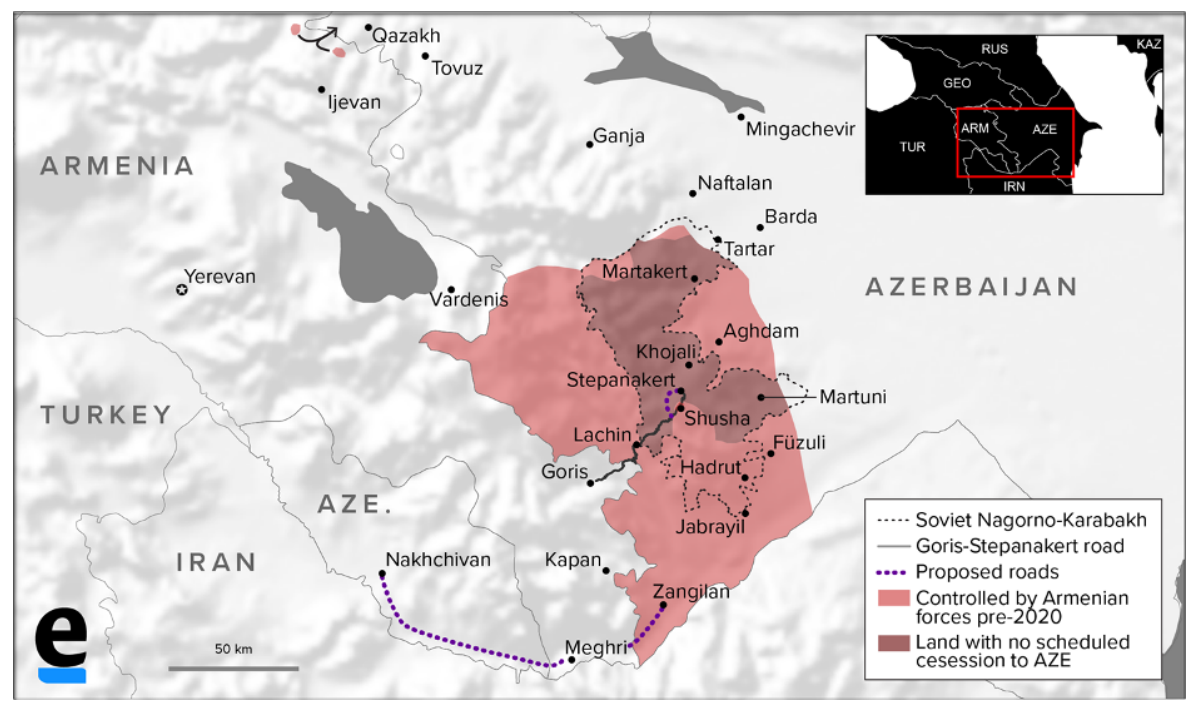

Abb. 1 Berg-Karabach nach dem Krieg (Quelle: Kucera 2020)

\section{Externe Akteure}

Über die Konfliktparteien hinaus wurde der Herbstkrieg 2020 sowie dessen Ausgang von zwei Schlüsselakteuren, nämlich Russland und der Türkei beeinflusst. Russland handelte den Deal vom 9. November in enger Abstimmung mit der Türkei aus. Dadurch demonstrierte Moskau nicht nur die eigene Macht, sondern auch die Machtlosigkeit der westlichen Akteure. Die Botschaft an Armenien und Aserbaidschan lautete, es führe kein Weg an Russland vorbei.

Die EU rief beim Ausbruch des Krieges zur Beendigung der Kampfhandlungen auf und verwies auf ihre Bereitschaft, die OSZE als Konfliktmediator zu unterstützen (Europäische Union 2020). Aber Brüssel war weder in der Lage, den Krieg in der unmittelbaren Nachbarschaft der EU zu verhindern, noch aktiv an dessen Beilegung teilzunehmen. Es wird deutlich, wie weit die EU vom Status eines geopolitischen Akteurs entfernt ist. Zwar versuchte Frankreich als Co-Vorsitzende der Minsk-Gruppe eine aktive Rolle einzunehmen, aber fokussierte dabei vor allem auf den Diskurs über die Türkei (Reuters 2020) als Bedrohung für die europäische Sicherheitsordnung. Die EU könnte sich in die Konfliktvermittlung möglicherweise aktiver einbringen, falls sie in der Minsk-Gruppe als Co-Vorsitzende Frankreich ersetzen würde, obwohl die Mink-Gruppe inzwischen massiv an Bedeutung verloren hat. Die USA, ein weiterer wichtiger Akteur in der Region und Co-Vorsitzende der Minsk-Gruppe, waren mit ihren Präsidentschaftswahlen beschäftigt, aber handelten am 25. Oktober eine humanitäre Waffenstillstandvereinbarung aus (U.S. Department of State 2020), die verletzt wurde.

Iran als unmittelbarer Nachbar der Konfliktparteien befürchtete einen SpilloverEffekt auf das iranische Territorium und damit eine Stärkung der separatistischen Bewegungen seitens der aserbaidschanischen Minderheit (18\% der Gesamtbevölke- 
rung). Aus diesem Grund verstärkte Iran die Stationierung der Truppen im Grenzgebiet und warb aktiv, aber ohne konkrete Ergebnisse mit dem eigenen Friedensplan zur Beilegung des Krieges.

Russland Obwohl Russland gemeinsam mit den anderen Co-Vorsitzenden der Minsk-Gruppe die Konfliktparteien direkt nach dem Ausbruch des Krieges zur Beendigung der Kampfhandlungen aufrief (Presidential Executive Office 2020b) und sich aktiv an den ausgehandelten Waffenstillstandvereinbarungen beteiligte, verzichtete Moskau auf eine aktive Einwirkung auf Aserbaidschan bzw. auf eine aktive militärische Unterstützung für den Bündnispartner Armenien. Die Rhetorik Aserbaidschans - ,Es ist ein Kampf zwischen uns und Armenien, und alle anderen sollten sich heraushalten“ (Alijew, zit. n. tagesschau 2020b) - sowie Armeniens - „Niemand versucht, sie aufzuhalten“ (Paschinjan, zit n. tagesschau 2020a) - deuten darauf hin, dass Aserbaidschan sich auf die Nichteinmischung seitens Moskaus verlassen konnte und Armenien sich vom Kreml hingehalten gefühlt hat.

Als am 31. Oktober der armenische Premierminister Paschinjan den russischen Präsidenten Wladimir Putin um dringende Hilfe zur Wahrung der Sicherheit Armeniens bat (News.am 2020), hatte das Land bereits große Verluste erlitten. In seiner Antwort sah sich der Kreml aber gemäß Schutzklausel der OVKS nicht in der Pflicht einzugreifen, bis sich die Kämpfe nicht auf das Territorium Armeniens verlagern würden (Außenministerium der Russischen Föderation 2020a). Russland mischte sich erst aktiv ein, als die Gefahr, dass auch Stepankert fallen würde, realistisch wurde. Durch den Erhalt von Stepankert, das nun von Aserbaidschan umgeben ist, ließ Moskau nicht zu, dass Aserbaidschan die Gesamtregion zurückerobert. Dadurch schaffte Russland die Notwendigkeit zur Stationierung von russischen Friedenstruppen in Berg-Karabach. Es ist anzunehmen, dass die russische Strategie im Allgemeinen drei Ziele verfolgte:

Erstens spielt Aserbaidschan als Partnerland eine wichtige Rolle. Denn Aserbaidschan ist ein strategisch und wirtschaftlich wichtiges Nachbarland an der hochexplosiven nordkaukasischen Grenze und am Kaspischen Meer. Außerdem ist Aserbaidschan aus der Sicht Moskaus ein beispielhaftes postsowjetisches Land, das nicht die EU- und NATO-Integration anstrebt und mit Russland gute Beziehungen pflegt. Der Faktor des steigenden geopolitischen Ungleichgewichts spielte ebenfalls eine entscheidende Rolle. Parallel zur aktiven Rüstungspolitik gab Aserbaidschan die Hoffnung auf eine internationale Konfliktvermittlung nach und nach auf und stützte sich stattdessen zunehmend auf die Türkei. Deshalb stand Russland vor dem Dilemma, entweder den Nutzen aus diesem Prozess zu ziehen (die längst angekündigte Stationierung von russischen Friedenstruppen), oder die Beziehungen zu Aserbaidschan und somit auch mit der Türkei zu gefährden.

Zweitens gab der Krieg Russland einen guten Anlass, anderthalb Jahre nach der Samtenen Revolution in Armenien, die Perspektive der Demokratisierung im Keim zu ersticken, seinen Einfluss auf Armenien zu verstärken und die Idee der Farbrevolution als gescheitert vorzuführen. Außerdem vereint aserbaidschanische und russische Eliten dieselbe Angst, dass Farbrevolutionen eine Gefahr für die autoritäre Machtsicherung darstellen. Denn aus diesem Grund ist Russland erst im Anschluss der gegen den Autoritarismus gerichteten Samtenen Revolution in Armenien bereit 
gewesen, die Balance zugunsten Aserbaidschans zu verändern. Es ist im Interesse Russlands, Paschinjan politisch zu vernichten, weil Paschinjan gegen die korrupten und mit Russland eng verbundenen armenischen Eliten (der sog. Karabach-Clan) für die Modernisierung Armeniens durch die Annäherung an den Westen steht. Er stellte zum Beispiel nicht nur die Mitgliedschaft Armeniens in der OVKS in Frage, sondern ging auch aktiv gegen den russischen Einfluss im Land vor.

Drittens konnte Moskau durch das Ergebnis des Krieges, trotz des offensichtlichen Eindringens der Türkei in seine exklusive Einflusszone, seinen Einfluss nicht nur auf Armenien, sondern auch auf Aserbaidschan verstärken. Russland toleriert die eingeschränkte Präsenz der Türkei, weil die Schwächung der Positionen des Westens nicht nur im Südkaukasus, sondern auch im Nahen Osten und in Nordafrika im beiderseitigen Interesse ist. Eine Vergrößerung der Wertekluft und geopolitischen Rivalität zwischen dem NATO-Mitglied Türkei und dem Westen ist eindeutig im Interesse der Russischen Föderation.

Türkei Neben der militärischen Überlegenheit war der Faktor Türkei entscheidend für den Erfolg Aserbaidschans. Die Türkei als strategischer Partner spielte die Rolle eines game-changers, indem sich Ankara pro-aktiv auf der Seite Bakus positionierte. Noch bis in den September führten die Türkei und Aserbaidschan eine gemeinsame Militärübung in der aserbaidschanischen Exklave Nachitschewan durch und damit vor der russisch-armenischen Militärübung Kavkaz-2020 in Armenien (Caucasuswatch 2020). Armenien warf Aserbaidschan den Einsatz ausländischer Söldner*innen mit türkischer Unterstützung vor. Medienberichte sahen den Vorwurf als bestätigt an, und er wurde von russischer Seite gestützt, die den sofortigen Abzug ausländischer Söldner*innen forderte (Außenministerium der Russischen Föderation 2020b). Die Vorwürfe wurden von der aserbaidschanischen Seite abgewiesen. Ein wichtiger Faktor für den Erfolg Aserbaidschans war die Präsenz türkischer F-16Kampfflugzeuge in der zweitgrößten Stadt Gandscha. Dadurch demonstrierte die Türkei die Verschiebung des geopolitischen Gleichgewichts zu Gunsten Aserbaidschans, was unter anderem die zurückhaltende Position Moskaus begründet. Im Hinblick auf die Vereinbarung vom 9. November fand offenbar zwischen türkischer und russischer Seite ein intensiver Austausch statt, in dessen Rahmen unter anderem die Zusammenarbeit in Syrien besprochen wurde (Presidency Of The Republic Of Turkey 2020).

In der Tat bestand das Hauptziel der Türkei darin, aus dem Krieg um Berg-Karabach als gestärkter Regionalakteur hervorzugehen und das gewonnene politische Kapital nicht nur im Südkaukasus, sondern auch im Nahen Osten oder im Mittelmeerraum gegenüber dem Westen und Russland einzusetzen. Darüber hinaus erhält die Türkei einen Zugang über den Landweg zum aserbaidschanischen Kernland und dadurch zu den Turkvölkern Zentralasiens.

\section{Fazit}

Am 9. November wurde zwar der Berg-Karabach-Krieg zwischen Armenien und Aserbaidschan beendet, aber nicht der Konflikt um Berg-Karabach, der aktuell wei- 
terbesteht. Der Krieg veränderte in erster Linie den zuvor bestehenden Status quo im Südkaukasus. Wie diese Veränderung zustande gekommen ist und wie ihre Zukunft aussehen wird, sollte für Besorgnis in Brüssel sorgen. Obwohl sich aufgrund der ineffektiven Konfliktvermittlung und Rüstungsspirale zwischen den Konfliktparteien der Ausbruch eines neuen Krieges in der unmittelbaren Nachbarschaft der EU abzeichnete, vermied es die EU, Verantwortung zu übernehmen und sich als regionaler Akteur zu profilieren. Russland führte die strategische Schwäche des Westens demonstrativ vor und teilte seine Einflusssphäre mit der Türkei. Dadurch demonstrierten Moskau und Ankara, wie sie von der Abwesenheit der EU und der USA nicht nur im Mittelmeerraum und im Nahen Osten, sondern auch im Südkaukasus profitieren können. Die EU wird sich im Hinblick auf ihre geopolitischen Interessen und transformative Kraft der Realität stellen müssen, dass das Ausmaß des russischen Einflusses auf Armenien, Aserbaidschan und allgemein auf den Südkaukasus größer wird.

Die EU wird zusehen müssen, wie der Kreml die demokratische Perspektive Armeniens, welches Teil der Östlichen Partnerschaft ist, rückgängig machen wird. Russland zog erfolgreich Nutzen aus der Tatsache, dass Armenien seit den 1990er Jahren eine Art Geisel der Berg-Karabach-Frage geworden war. Jetzt ist es nur eine Frage der Zeit, bis der armenische Premierminister Nikol Paschinjan von einem moskautreuen Premierminister aus dem Amt gedrängt wird. Gleichzeitig verhalf Moskau Baku indirekt bei der unvollendeten Wiederherstellung der territorialen Integrität und erhielt die Möglichkeit eigene Streitkräfte wieder auf dem aserbaidschanischen Territorium zu stationieren. Letztendlich fungiert seit dem 9. November nicht mehr Armenien, sondern Russland als Schutzpatron Berg-Karabachs. Den dafür erforderlichen Preis mit bitterem Beigeschmack wird Aserbaidschan allerdings noch zahlen müssen, weil russische Friedenstruppen erfahrungsgemäß nicht unbedingt dem Frieden dienen.

Funding Open Access funding enabled and organized by Projekt DEAL.

Open Access Dieser Artikel wird unter der Creative Commons Namensnennung 4.0 International Lizenz veröffentlicht, welche die Nutzung, Vervielfältigung, Bearbeitung, Verbreitung und Wiedergabe in jeglichem Medium und Format erlaubt, sofern Sie den/die ursprünglichen Autor(en) und die Quelle ordnungsgemäß nennen, einen Link zur Creative Commons Lizenz beifügen und angeben, ob Änderungen vorgenommen wurden.

Die in diesem Artikel enthaltenen Bilder und sonstiges Drittmaterial unterliegen ebenfalls der genannten Creative Commons Lizenz, sofern sich aus der Abbildungslegende nichts anderes ergibt. Sofern das betreffende Material nicht unter der genannten Creative Commons Lizenz steht und die betreffende Handlung nicht nach gesetzlichen Vorschriften erlaubt ist, ist für die oben aufgeführten Weiterverwendungen des Materials die Einwilligung des jeweiligen Rechteinhabers einzuholen.

Weitere Details zur Lizenz entnehmen Sie bitte der Lizenzinformation auf http://creativecommons.org/ licenses/by/4.0/deed.de. 


\section{Literatur}

\section{Verwendete Literatur}

Aliyev, I. (2019). Ilham Aliyev was interviewed by Rossiya-24 TV channel. https://en.president.az/articles/ 35459. Zugegriffen: 30. Okt. 2020.

Amnesty International (2020). Armenia/Azerbaijan: First confirmed use of cluster munitions by Armenia 'cruel and reckless'. https://www.amnesty.org/en/latest/news/2020/10/armenia-azerbaijan-firstconfirmed-use-of-cluster-munitions-by-armenia-cruel-and-reckless/. Zugegriffen: 11. Nov. 2020.

Außenministerium der Russischen Föderation (2020a). Foreign Ministry statement in connection with Prime Minister of Armenia Nikol Pashinyan's address to President of Russia Vladimir Putin. https:// www.mid.ru/en/press_service/spokesman/official_statement/-/asset_publisher/t2GCdmD8RNIr/ content/id/4411939. Zugegriffen: 1. Nov. 2020.

Außenministerium der Russischen Föderation (2020b). О телефонных разговорах Министра иностранных дел России С.В.Лаврова с Министром иностранных дел Азербайджана Д.А.Байрамовым и Министром иностранных дел Армении 3.Г.Мнацаканяном [Zu den Telefongesprächen des russischen Außenministers mit seinen armenischen und aserbaidschanischen Kollegen]. https://www.mid.ru/ru/foreign_policy/news/-/asset_publisher/cKNonkJE02Bw/ content/id/4363752. Zugegriffen: 10. Nov. 2020.

BBC (2020). Nagorno-Karabakh conflict killed 5,000 soldiers. https://www.bbc.com/news/world-europe55174211. Zugegriffen: 10. Dez. 2020.

Caucasuswatch (2020, 8. Sept.). Azerbaijan and Turkey conduct large military exercise in Nakhchivan. https://caucasuswatch.de/news/3079.html. Zugegriffen: 3. Nov. 2020.

De Waal, T. (2011). The Caucasus: An Introduction. Oxford: Oxford University Press.

De Waal, T. (2020). Europe's longest-running conflict can't be ignored. Carnegie Moscow Center. https:// carnegie.ru/commentary/82813. Zugegriffen: 1. Nov. 2020.

Europäische Union (2020). Nagorno-Karabakh: Declaration by the High Representative on behalf of the European Union. https:/www.consilium.europa.eu/en/press/press-releases/2020/10/11/nagornokarabakh-declaration-by-the-high-representative-on-behalf-of-the-european-union/. Zugegriffen: 1. Nov. 2020

Halbach, U. (2020a). Nagorny-Karabach. Bundeszentrale für politische Bildung (bpb). https://www.bpb. de/internationales/weltweit/innerstaatliche-konflikte/224129/nagorny-karabach. Zugegriffen: 10. Nov. 2020.

Halbach, U. (2020b). Neuere Entwicklungen im Konflikt zwischen Armenien und Aserbaidschan. SWPAktuell 2020/A71.1. https://www.swp-berlin.org/fileadmin/contents/products/aktuel1/2020A71_ armenien_aserbaidschan.pdf.Zugegriffen. Zugegriffen: 30. Nov. 2020.

Human Rights Watch (2020). Azerbaijan: Cluster munitions used in Nagorno-Karabakh. https://www.hrw. org/news/2020/10/23/azerbaijan-cluster-munitions-used-nagorno-karabakh. Zugegriffen: 11. Nov. 2020.

International Crisis Group (2007). Nagorno-Karabakh. Risking war. https://www.files.ethz.ch/isn/45472/ 187_nagorno_karabakh_risking_war.pdf. Zugegriffen: 7. Nov. 2020.

International Crisis Group (2020). The Nagorno-Karabakh conflict: A visual explainer. https://www. crisisgroup.org/tr/node/12543. Zugegriffen: 7. Nov. 2020.

Kucera, J. (2019, 6. Aug.). Pashinyan calls for unification between Armenia and Karabakh. Eurasianet. https://eurasianet.org/pashinyan-calls-for-unification-between-armenia-and-karabakh. Zugegriffen: 7. Nov. 2020.

Kucera, J. (2020, 11. Nov.). In Karabakh deal, as many questions as answers. Eurasianet. https://eurasianet. org/in-karabakh-deal-as-many-questions-as-answers. Zugegriffen: 11. Nov. 2020.

Mediamax (2019, 8. Apr.). Davit Tonoyan: People need to value our victory. https://mediamax.am/en/news/ interviews/33013/?fbclid=IwAR0sDbyRrD959O8ffTOJpdTh9wyY1bY0_QwLwOasCN7zLIJ0ow7 3G7Aq6yQ. Zugegriffen: 3. Nov. 2020.

Meister, S. (2020). Nicht ganz so eingefroren. Internationale Politik, 75(3), 10-12.

Mutschler, M., \& Bales, M. (2019). Globaler Militarisierungsindex 2019. Bonn International Center for Conversion (BICC). https://www.bicc.de/uploads/tx_bicctools/BICC_GMI_2019_D.pdf. Zugegriffen: 3. Nov. 2020.

NEWS.am (2020, 31. Okt.). Armenia's Pashinyan sends letter to Russia's Putin to determine types, amount of assistance. https://news.am/eng/news/610760.html. Zugegriffen: 8. Nov. 2020. 
OSZE (2009). Statement by the OSCE Minsk Group Co-Chair countries. https://www.osce.org/mg/51152. Zugegriffen: 4. Nov. 2020.

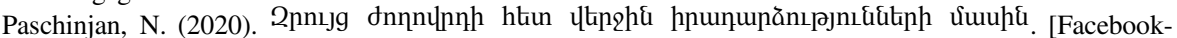
live des armenischen Premierministers über die Vereinbarung vom 9. November]. https://www. facebook.com/nikol.pashinyan/videos/726655837939351/. Zugegriffen: 10. Nov. 2020.

Presidency Of The Republic Of Turkey (2020). Phone call with President Vladimir Putin of Russia. https:// www.tccb.gov.tr/en/speeches-statements/558/122748/phone-call-with-president-vladimir-putin-ofrussia. Zugegriffen: 11. Nov. 2020.

Presidential Executive Office (2020a). Statement by President of the Republic of Azerbaijan, Prime Minister of the Republic of Armenia and President of the Russian Federation. http://en.kremlin.ru/acts/ news/64384. Zugegriffen: 10. Nov. 2020.

Presidential Executive Office (2020b). Statement of the presidents of Russia, the United States and France on Nagorno-Karabakh. http://en.kremlin.ru/events/president/news/64133. Zugegriffen: 2. Okt. 2020.

Quiring, M. (2009). Schwelende Konflikte in der Kaukasus-Region. Aus Politik und Zeitgeschichte (APuZ), (13), 19.

Reuters (2020, 30. Sept.). Macron criticises Turkey’s "warlike” rhetoric on Nagorno-Karabakh. https:// www.reuters.com/article/us-armenia-azerbaijan-france-idUSKBN26L19R. Zugegriffen: 3. Nov. 2020.

Safi, M. (2020, 28. Sept.). Dozens killed as Armenian and Azerbaijani forces clash for second day. The Guardian. https://www.theguardian.com/world/2020/sep/28/dozens-dead-as-armenia-azerbaijanclashes-continue. Zugegriffen: 14. Nov. 2020.

SIPRI - Stockholm International Peace Research Institute (2019). Data for all countries 1949-2019. https:// www.sipri.org/databases/milex. Zugegriffen: 3. Nov. 2020.

SIPRI (2020). Trends in international arms transfer 2019. https://www.sipri.org/sites/default/files/2020-03/ fs_2003_at_2019.pdf. Zugegriffen: 3. Nov. 2020.

Smolnik, F., \& Halbach, U. (2016). Der Konflikt um Berg-Karabach im Lichte der Krise um die Ukraine. Stiftung Wissenschaft und Politik (SWP). https://www.swp-berlin.org/fileadmin/contents/products/ studien/2016S13_fhs.pdf. Zugegriffen: 8. Nov. 2020.

Stöber, S. (2020a, 13. Okt.). Konflikt ohne Lösung. tagesschau.de. https://www.tagesschau.de/ausland/ bergkarabach-armenien-aserbaidschan-101.html. Zugegriffen: 7. Nov. 2020.

Stöber, S. (2020b, 6. Okt.). Neue Waffen verändern den Krieg. tagesschau.de. https://www.tagesschau.de/ ausland/bergkarabach-waffen-drohnen-raketen-101.html. Zugegriffen: 30. Okt. 2020.

Tagesschau (2020a, 7. Nov.). Krieg um Bergkarabach. „Niemand versucht, sie aufzuhalten“. tagesschau.de. https://www.tagesschau.de/ausland/bergkarabach-armenien-paschinjan-101.html. Zugegriffen: 10. Nov. 2020.

Tagesschau (2020b, 7. Nov.). Aserbaidschans Präsident. „Ein Kampf zwischen uns und Armenien“. tagesschau.de. https://www.tagesschau.de/ausland/aserbaidschan-aliyev-101.html. Zugegriffen: 8. Nov. 2020.

U.S. Department of State (2020). U.S.-Armenia-Azerbaijan Joint Statement. https://www.state.gov/u-sarmenia-azerbaijan-joint-statement/. Zugegriffen: 28. Okt. 2020.

UNICEF (2020). UNICEF statement on one month of fighting in and beyond Nagorno-Karabakh. https:// www.unicef.org/armenia/en/press-releases/unicef-statement-one-month-fighting-and-beyond-nagor no-karabakh. Zugegriffen: 10. Nov. 2020.

Zeit Online (2020, 4. Nov.). Aserbaidschan nimmt mehrere Orte in Bergkarabach ein. https://www.zeit. de/politik/ausland/2020-11/suedkaukasus-bergkarabach-aserbaidschan-raketen-armenien-konfliktniederlage. Zugegriffen: 4. Nov. 2020.

\section{Weiterführende Literatur}

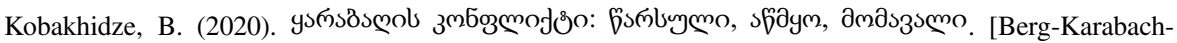
Konflikt: Vergangenheit, Gegenwart und Zukunft]. https://publika.ge/article/yarabaghis-konfliqtiwarsuli-awmyo-momavali/. Zugegriffen: 29. Okt. 2020. 